\title{
Automatic Vehicle License Plate Detection using K-Means Clustering Algorithm and CNN
}

\author{
Dr. Joy Iong Zong Chen, \\ Professor, \\ Department of Electrical Engineering, \\ Da-Yeh University, \\ Taiwan. \\ Email id: jchen@mail.dyu.edu.tw
}

\begin{abstract}
Because of the development of highways as well as the increased number of vehicles usage, much attention is required on to develop an efficient and safe intelligent transportation system. The aspect of identifying specific objects present in an image is an important criteria in areas like digital image processing and computer vision. Because of the different formats, colours, shapes, viewpoints and non-uniform illumination environment of license plates, recognising the same proves to be a tasking issue. In this paper, we present a vehicle license plate recognition model using convolutional neural network $(\mathrm{CNN})$ and $\mathrm{K}$ means clustering based segmentation. This methodology works on three major steps such as detection and segmentation using K-means clustering and recognition of the number in the license plate using $\mathrm{CNN}$ model. We have also used location and detection algorithms to improve the accuracy of detection. The experimental investigation is carried out using datasets and the observed simulation results prove that the proposed mode is more effective than the other methodologies introduced so far.
\end{abstract}

Keywords: license plate detection; intelligent transportation system; convolutional neural network; character recognition

\section{Introduction}

In recent years, there has been quick progression in Graphical Processing Units (GPU) and Intelligent Transportation System (ITS) and License plate detection and recognition. This has attracted research interests in multiple domains. License Plate Recognition (LPR) has a number of applications such as traffic safety administration, security management and unmanned parking fields. However, because of the distinct format of the plates that vary 
Journal of Electrical Engineering and Automation (EEA) (2021)

Vol.03/ No.01

Pages: $15-23$

https://www.irojournals.com/iroeea

DOI: https://doi.org/10.36548/jeea.2021.1.002

according to various criteria like distance between vehicle and camera, vehicles speed, brightness, background, namely and image acquisition. Due to these considerations, there are a number of restrictions like static background, allocated paths, lower vehicle speed and permanent illumination that are required to capture the license plate and identify the characters [1]. There are four common blocks that are used in an LPR technique namely character standardization and classification, segmentation, LP localization and vehicle image. However, because of it is tedious to locate the plate, this process is said to be complex and will also have a direct impact on the efficiency and accuracy of the consecutive steps. Hence, it is crucial that the issues due to background disturbances and illumination conditions do not exist [2]. A number of researchers and inventers have proposed many approaches such as line sensitive filters and edge prediction approaches to extract details of arithmetic morphology, window scheme and plate region. Though a number of models proposed so far are capable of determining the position of LP, it has a number of disadvantages such as absence versatility, higher computation time and sensitivity to illumination when used on a diverse platform [3]. By linking components, morphology and relaxation label, character segmentation is made possible. Moreover, it also comprises of maximum count of character analyzing techniques such as K-nearest neighbour [4], Markov chain model [5], support vector machine, fuzzy cmeans, Artificial Neural Networks (ANN) [6] and Baye's classification. Though it is possible to calculate the aspect of using LP analysis and segmentation, many models are capable of processing only 2 types of character analysis and individual line character segmentation. As far as LPR is concerned, it works on character recognition and segmentation. Hence placement of LP is deemed to be exhaustive and extensive segregation of a single part in order to combine the images to extract concrete information.

In this paper, we have used a VLPR using DL model with K-means clustering and CNN algorithm. This methodology works on three major steps such as detection and segmentation using K-means clustering and recognition of the number in the license plate using CNN model. We have also used location and detection algorithms to improve the accuracy of detection. The experimental analysis of this methodology takes place using three datasets and a comparative study is made on the results obtained. Section 2 gives a survey analysis on similar methodologies and section 3 outlines the proposed work. Experimental analysis is performed in section 4 and accordingly a conclusion is drawn in section 5 . 


\section{Related Works}

In [7] the authors have developed a novel technique that can be used to identify the LPs. This will enable identification of vehicles and will also decrease the plate prediction false positives by a drastic measure. Using CNN has significantly improved character recognition value even in noisy and blurred images. Researchers in [8] have concentrated on using morphological attributes to predict the License Plate. This model has the ability to identify the different alphabets in the English language. Similarly, in [x], the authors have analyzed the use of optical character recognition using correlation technique and templates mapping for plate detection, in an automatic manner. In [9] a 4 step process for analyzing LP is proposed. As the first step, the images that are pre-processed are obtained from cameras which are then adjusted to the apt brightness. Noise present is eliminated and then converted into grey scale image. The LP location is extracted using the edge of the images used. Moreover, segmentation of the LP is done using the characters and they could be used to identify every character using template matching methodology. A number of detecting algorithms have been analyzed in [10] and the relationship between them is analyzed. This methodology is also applied using Euclidean distance transformation and CCA. Therefore, these models are highly affected by improper illumination and can provide better accuracy under normal circumstances. In [11], wavelet Laplacian technique is used for separating the different characters that are obtained from the video image. In this methodology, the zero crossing points are identified in order to determine the space between the letters. However, if there is a backdrop in the license plate, it might affect the identification of characters. Moreover, in [12] the image that is used as the input has binarized due to the presence among characters and absence of character shapes. Thus the images which comprise of a complicated backdrop will require a more profound binarization model which can differentiate between the background data and foreground data. A number of researches have also been conducted to remove the impact of less illumination which has other effects on difficult backgrounds and blurred images [13].

\section{Development of Use Case Scenario}

Fig.1 shows the working of the proposed hybrid LP identification method. At the initial stage, the recognition and localization of LP is done using CCA and IBA model. This 
is followed by segmentation of LP with the help of K-Means Clustering. Finally, for character recognition, $\mathrm{CNN}$ is used to detect and recognise the characters that occur in an LP.

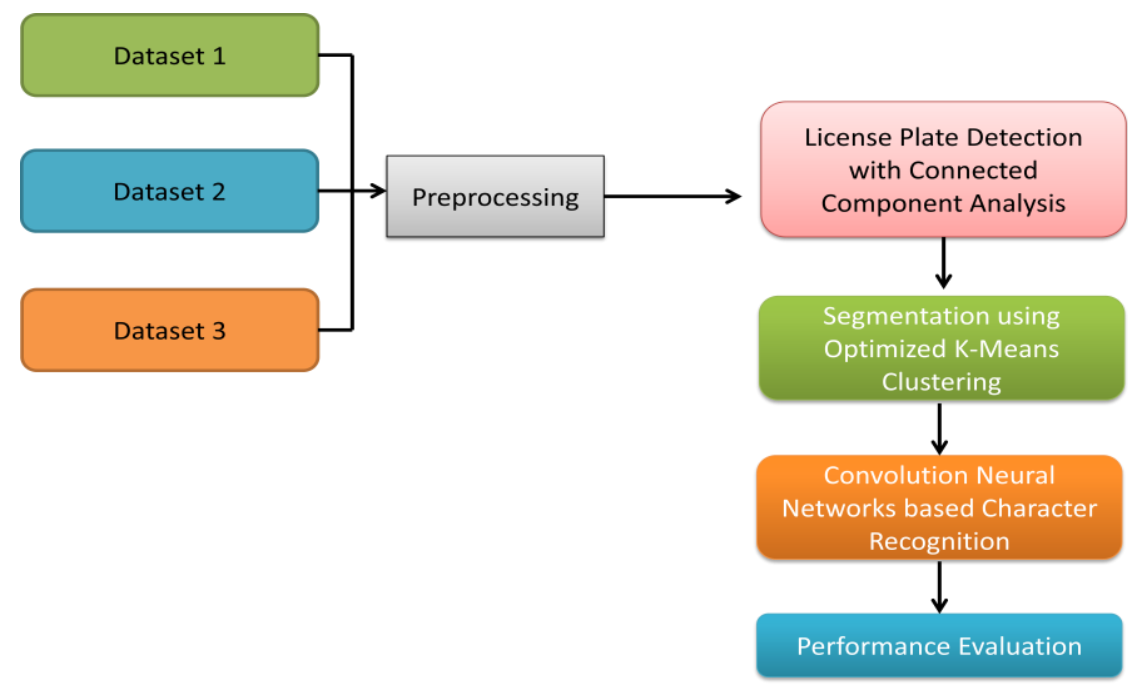

Fig.1. Framework of the Proposed K-Means and CNN model

\subsection{Localization and Recognition Process}

Depending on the rule of the plate as well as the lighting effect on the plate, the dissemination of different location of the image will differ. Since it is not possible to produce the required output using binary model, local binary techniques are used. The local binary techniques are initially classified into $\mathrm{m} \times \mathrm{n}$ blocks in an image such that every block that is split is calculated with binary model. IN the proposed methodology, we have used two binary methodologies that can be used on the sub-blocks. Hence, otsu function is applicable on illumination constraints which has high variation. On the other hand, in order to overcome the barrier of irregular illumination, we have used enhanced BA for eliminating the shadow. Consider that a typical block consists of point $(x, y)$ of $f(x, y)$ which represents the gray value. The threshold value can be expressed as follows:

$$
T(x, y)=\frac{-w \leq z, 1 \leq w f(x+1, y+z) \pm w \leq z, 1 \leq w f(x+1, y+z)}{2}
$$


Journal of Electrical Engineering and Automation (EEA) (2021)

Vol.03/ No.01

Pages: $15-23$

https://www.irojournals.com/iroeea

DOI: https://doi.org/10.36548/jeea.2021.1.002

Accordingly, the binary image can be determined as follows:

$$
b(x, y)= \begin{cases}0, \text { if } f(x, y)<T(x x, y) \\ 255, & \text { otherwise }\end{cases}
$$

where $s=(2 w+1)$ is the window size of the block. Using this methodology, conservation of the characters as well as elimination of noise is very efficient using this methodology.

\subsection{Character Segmentation}

To determine the uncertainty of a dataset, neutrosophic analysis plays a crucial part. The

Truth (TR), indeterminacy (I) and falsity (F) are used to identify the degree of certainty. In $\mathrm{A}(\mathrm{x}, \mathrm{y})$ which is the pixel, it can be identified that

$$
A(t, p f,)=\{F(x, y), I(x, y), T R(x, y)\}
$$

If the local means value is denoted by $\bar{A}(\mathrm{x}, \mathrm{y})$ for pixel $(\mathrm{x}, \mathrm{y})$, then the membership function can be determined using:

$$
\begin{aligned}
& T R(x, y)=\frac{\bar{A}(x, y)-\bar{A}}{A-\bar{A}} \\
& F(x, y)=1-\operatorname{TR}(x, y)
\end{aligned}
$$

\subsection{K-Means Clustering Algorithm}

This methodology is used to gather the objects using clustering method, into blocks and can be expressed arithmetically as follows:

$$
K=\sum_{u=1}^{u} \sum_{v=1}^{d u}\left\|X_{v}-Z_{u}\right\|
$$


DOI: https://doi.org/10.36548/jeea.2021.1.002

Where $u$ represents the count of clusters du denotes the pixel number and $Z_{u}$ represents the $\mathrm{u}^{\text {th }}$ cluster. Fig. 2 represents the different layers of character segmentation and recognition involved in the proposed methodology.

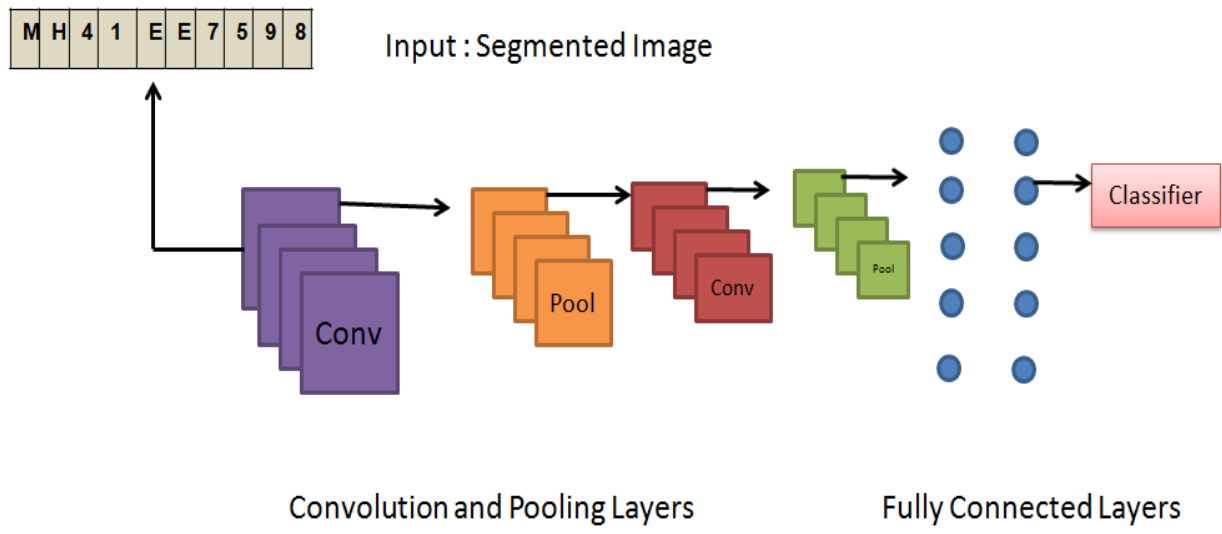

Fig.2. Character Segmentation and Recognition

\section{Results and Discussion}

Fig.3 shows the analysis of the proposed hybrid methodology with other methodologies comparing Map, F-score, recall and precision. It is observed that similar to analysis with dataset of FZU cars, a dataset of Stanford was also analysed using the proposed work in Fig.4. and it was identified that it was able to detect the characters upto $89 \%$ which is a significant improvement over the previous existing work.

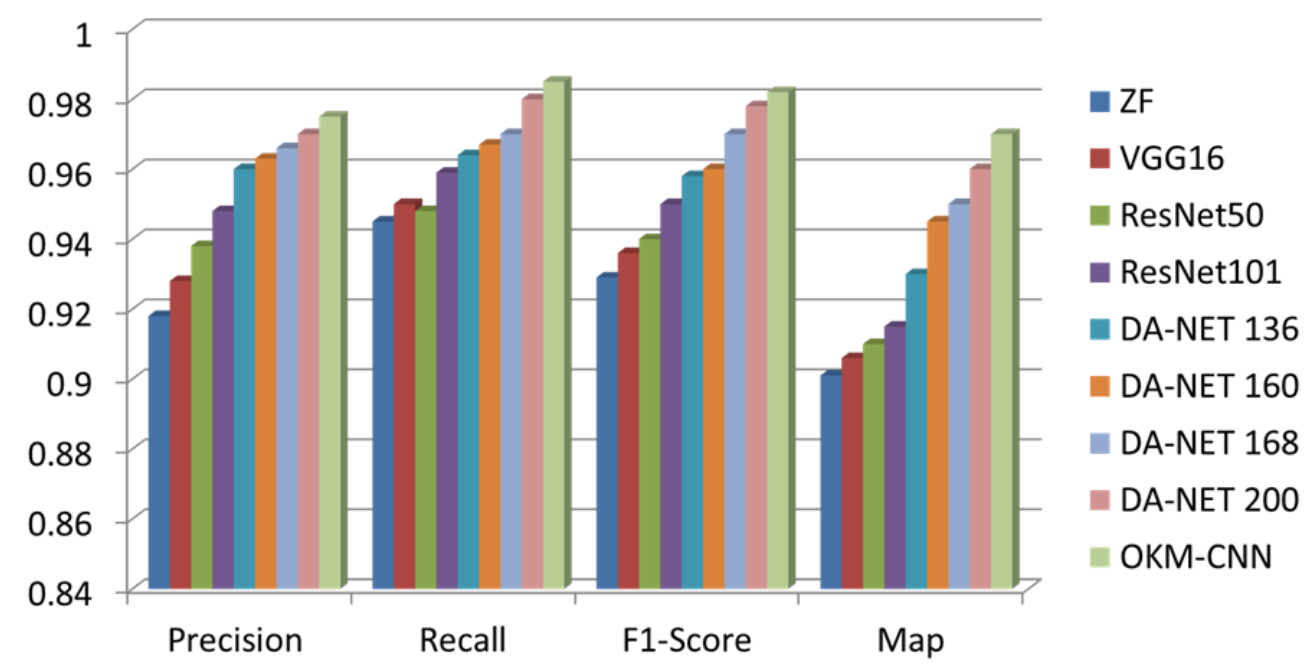

Fig.3. Dataset of F1-Score Result Analysis 
Journal of Electrical Engineering and Automation (EEA) (2021)

Vol.03/ No.01

Pages: 15-23

https://www.irojournals.com/iroeea

DOI: https://doi.org/10.36548/jeea.2021.1.002

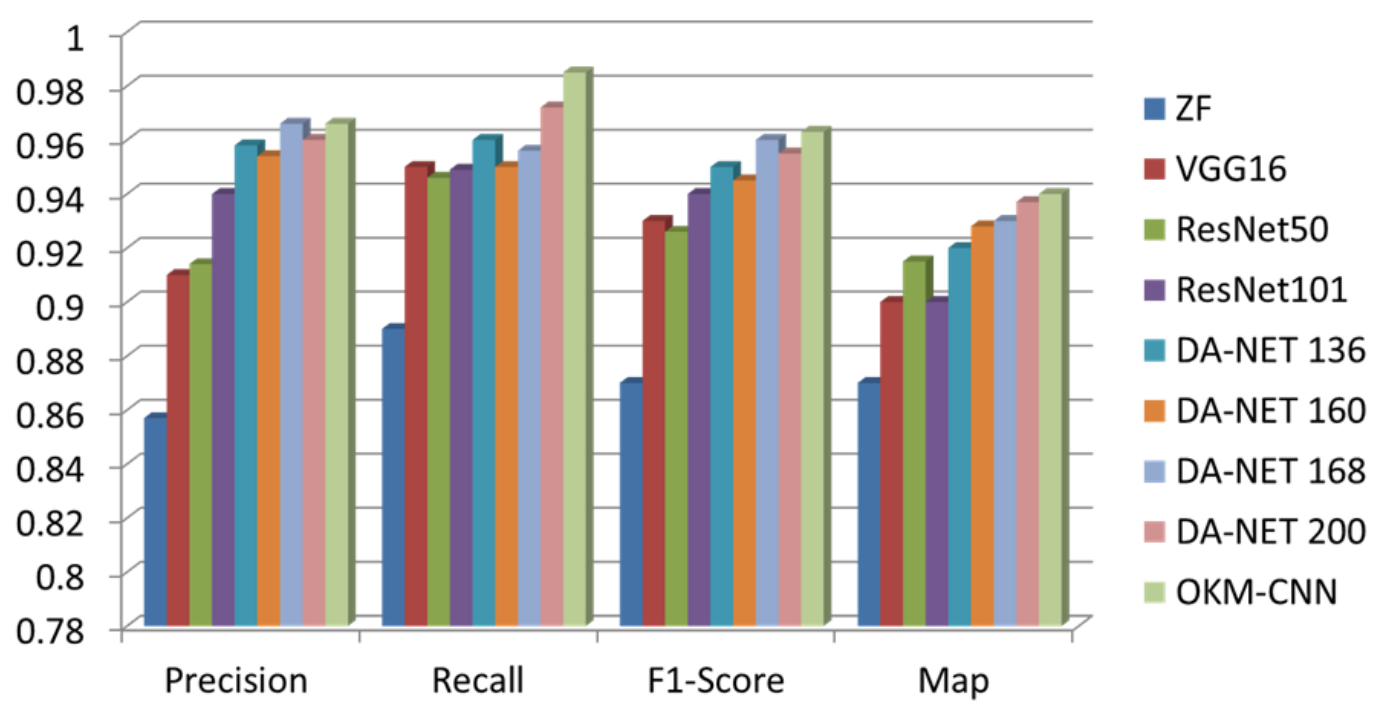

Fig.4. Dataset of Stanford Result Analysis

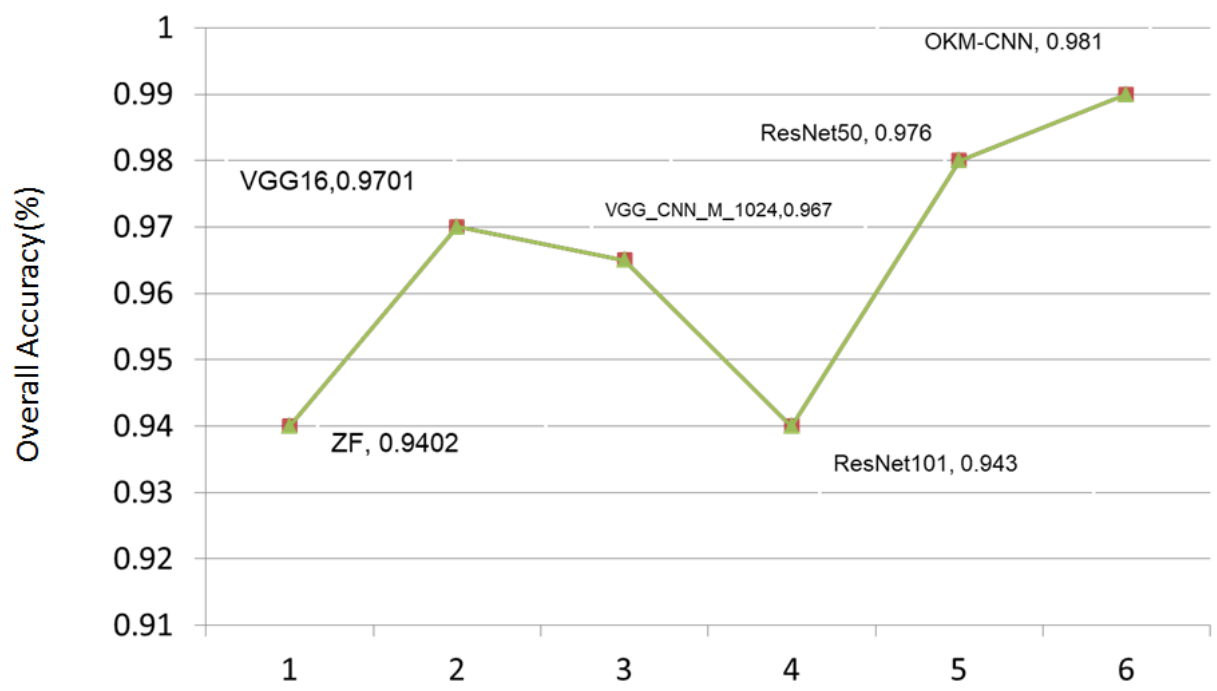

Fig.5. Overall Analysis of the Different Models

The accuracy analysis of the entire proposed work can be summarised in Fig.5 where the proposed methodology and that of the applied models for comparative analysis. It has been identified that the accuracy of this work is $98.1 \%$ more efficient than the rest of the methodologies that are used for comparison. 


\section{Conclusion}

In this paper, we have represented a novel methodology that involves convolutional neural networks and k-means clustering to effectively recognize and detect license plates in the vehicles. There are three main steps involved in the proposed work. The first step involves detection and localization of the LP with the help of CCA and IBA model. In the next step, clustering methodology is incorporated to segment the LP images and in the last step, recognition of the characteristics takes place with the help of CNN model. The proposed model can be used as the central form of intelligent infrastructure like traffic surveillance, parking management and toll fee collection. Three sets of data are used in this methodology to determine the effectiveness of the proposed work. It has been observed that this methodology provides a more accurate value when compared with other similar models and can also be extended to identifying LPs in different languages.

\section{References}

[1] Silva, S. M., \& Jung, C. R. (2020). Real-time license plate detection and recognition using deep convolutional neural networks. Journal of Visual Communication and Image Representation, 71, 102773.

[2] Zheng, D., Zhao, Y., \& Wang, J. (2005). An efficient method of license plate location. Pattern recognition letters, 26(15), 2431-2438.

[3] Du, S., Ibrahim, M., Shehata, M., \& Badawy, W. (2012). Automatic license plate recognition (ALPR): A state-of-the-art review. IEEE Transactions on circuits and systems for video technology, 23(2), 311-325.

[4] Hsu, G. S., Chen, J. C., \& Chung, Y. Z. (2012). Application-oriented license plate recognition. IEEE transactions on vehicular technology, 62(2), 552-561.

[5] Bashar, D. A. (2020). Review on sustainable green Internet of Things and its application. J. Sustain. Wireless Syst, 1(4), 256-264.

[6] Parker, J. R., \& Federl, P. (1996). An approach to license plate recognition.

[7] Shirley, D. R. A. (2014, July). Systematic diagnosis of power switches. In 2014 International Conference on Embedded Systems (ICES) (pp. 32-34). IEEE.

[8] Xu, Z., Yang, W., Meng, A., Lu, N., Huang, H., Ying, C., \& Huang, L. (2018). Towards end-to-end license plate detection and recognition: A large dataset and 
Journal of Electrical Engineering and Automation (EEA) (2021)

Vol.03/ No.01

Pages: $15-23$

https://www.irojournals.com/iroeea

DOI: https://doi.org/10.36548/jeea.2021.1.002

baseline. In Proceedings of the European conference on computer vision (ECCV) (pp. 255-271).

[9] Lin, C. H., Lin, Y. S., \& Liu, W. C. (2018, April). An efficient license plate recognition system using convolution neural networks. In 2018 IEEE International Conference on Applied System Invention (ICASI) (pp. 224-227). IEEE.

[10] Zhang, L., Wang, P., Li, H., Li, Z., Shen, C., \& Zhang, Y. (2020). A robust attentional framework for license plate recognition in the wild. IEEE Transactions on Intelligent Transportation Systems.

[11] Adithya, M., Scholar, P. G., \& Shanthini, B. (2020). Security Analysis and Preserving Block-Level Data DE-duplication in Cloud Storage Services. Journal of trends in Computer Science and Smart technology (TCSST), 2(02), 120-126.

[12] Pustokhina, I. V., Pustokhin, D. A., Rodrigues, J. J., Gupta, D., Khanna, A., Shankar, K., ... \& Joshi, G. P. (2020). Automatic vehicle license plate recognition using optimal K-means with convolutional neural network for intelligent transportation systems. IEEE Access, 8, 92907-92917.

[13] Smys, S., Wang, H., \& Basar, A. (2021). 5G Network Simulation in Smart Cities using Neural Network Algorithm. Journal of Artificial Intelligence, 3(01), 4352. 